\title{
Theoretical study of electronic transport through a small quantum dot with a magnetic impurity
}

\author{
Mugurel Tुolea \\ National Institute of Materials Physics, POBox MG7, Bucharest-Magurele, Romania and \\ Institute of Molecular Physics, Polish Academy of Science, \\ ul. M. Smoluchowskiego 17, 60-179 Poznań, Poland \\ Bogdan R. Bułka \\ Institute of Molecular Physics, Polish Academy of Science, \\ ul. M. Smoluchowskiego 17, 60-179 Poznań, Poland
}

\begin{abstract}
We model a small quantum dot with a magnetic impurity by the Anderson Hamiltonian with a supplementary exchange interaction term. The transport calculations are performed by means of the Green functions within the equation of motion scheme, in which two decoupling procedures are proposed, for high and low temperatures, respectively. The paper focuses on the charge fluctuations for such a system, aspect not addressed before, as well as on the Kondo resonance. We show a specific role of the excited state, which can be observed in transport and in spin-spin correlations. Our studies show on a new many-body feature of the phase shift of transmitted electrons, which is manifested in a specific dip. In the Kondo regime, our calculations complement existing theoretical results. The system shows three Kondo peaks in the density of states: one at the Fermi energy and two side peaks, at a distance corresponding to the singlet-triplet level spacing. The existence of the central peak is conditioned by a degenerate state (the triplet) below the Fermi energy.
\end{abstract}

\section{INTRODUCTION}

Quantum dots (QD) are promising systems for nano-technology because their quantum-mechanical parameters are easy to control. Motivated, in part, by the recent developing in the field of magnetic semiconductors [1], or by the spintronic advances, theorists and experimentalists have started to address also the problem of quantum dots with magnetic impurities [2, 3, 4, 5, 6, 7]. In such systems, electrons in the quantum dot are subject to a supplementary exchange interaction. This induces a competition between the formation of the Kondo cloud and the magnetic interactions inside QD, but also specific charge fluctuations, aspect less addressed in literature. Sometimes, the role of the impurity is played by another quantum dot with odd occupancy [8], but also, as in a recent experiment [5], the impurity can be a transitional magnetic atom (Co). The splitting of the Kondo peak was attributed by the authors to the indirect RKKY interaction 9, 10, 11]. We believe that a central peak would have also been present for a much lower temperature. Govorov [2] studied theoretically the spectrum and the response to optical excitations of an InGaAs/GaAs quantum dot with a single Mn impurity. Recently, Murthy [3] also addressed the problem of interplay between the Stoner and the Kondo effect in a large QD with a magnetic impurity.

The competition between the Kondo and antiferromagnetic coupling was also intensively studied in a double-QD geometry coupled by hopping or by the RKKY interaction (e.g. 9, 10, 11, 12, 13, 14, 15, 16, 17, 18]). This is related with another interesting issue: the Kondo effect in QD with an integer spin [19, 20, 21, 22, 23]. In the experiment of Sasaki [23], the applied magnetic field can change the ground state and the relative position of the singlet and triplet states, and it can also induce a degeneracy point in the energy spectrum. In the vicinity of the degeneracy point, three Kondo peaks are noticed in the differential conductance, which is in agreement with our calculations for the Kondo regime.

Previous theoretical papers have focused mainly on the Kondo effect in large QDs with magnetic impurities, or on the two-impurity problem. In the present paper we are also interested in the Kondo resonance, but in a different system: a small QD with a magnetic impurity that is coupled only to the electrons in the dot. We are interested in interplay between magnetic interactions: the dot-leads Kondo coupling (as in the experimental set-up [24, 25]) and the intra-dot magnetic exchange interactions (between electrons in QD and the magnetic impurity). We would like to consider spin-flip processes on the impurity and their detection by the current flowing through the QD, both in high and low temperature regimes. In spectral terms, the flip of the impurity spin is associated with singlet-triplet excitations. To our knowledge, the problem was not considered yet.

Apart from the Kondo regime, we shall also analyze the regime of high temperatures. The solution in this case is more easy to obtain, and it allows simple analytical expressions. These will be used to discuss the charge fluctuations and spin-spin correlations aspects. An electron that enters QD forms with the magnetic impurity a correlated singlet or a triplet ground state, respectively to the sign of the exchange parameter. Both situations are met in experiments [5]. In these cases, the excited states also play an important role. It can be seen in the conductance peaks, which 


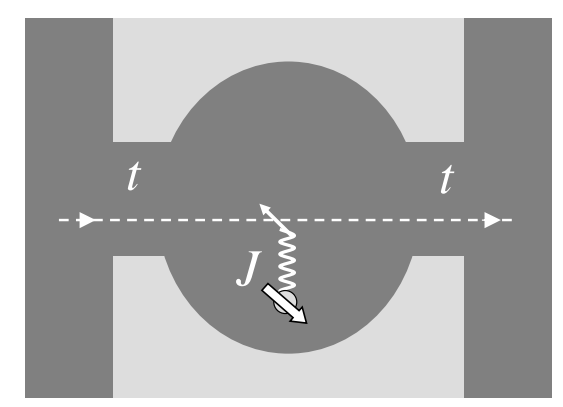

FIG. 1: Scheme of a quantum dot coupled to ideal leads through tunnelling barriers $t$. A magnetic impurity interacts with electrons in the dot through an exchange coupling $J$.

amplitude strongly depend on the coupling to the leads, and in the spin-spin correlation functions, especially when the excited states lie below the Fermi level. We also address the problem of the transmission phase. The measurement of the transmission phase (and not only the amplitude) is possible to realize, for some time [26, 27], and can provide interesting supplementary information. For our system, a dip can be noticed in the phase evolution, precisely at the excited state position, in condition of constant electronic occupancy on QD. We will discuss our results in connection with a recent experiment 28], where similar features were observed. Our studies are an explicit demonstration of a generalized Fridel sum rule in the presence of electronic correlations, the theorem which was just recently proved by Rontani [29].

The paper is organized as follows: in the Section II and III we describe our model and the Green functions approach. The equation of motion (EOM) technique is presented in detail. A decoupling scheme adequate for high temperatures is presented in section IV. The scheme allows to obtain analytical results for the conductance, dot occupancy, spin-spin correlator and the phase evolution. In Section V, we get an insight into the Kondo regime using a more advanced decoupling scheme. One can observe the formation of three Kondo peaks in the local DOS: at the Fermi energy and two side peaks. The central peak exists only if a degenerate state (the triplet) is below the Fermi energy. Section VI contains concluding remarks. For completeness, the spectrum of the system and a discussion on the formation of the Kondo resonance are presented in the Appendix A and B, respectively.

\section{MODEL OF A QUANTUM DOT WITH A MAGNETIC IMPURITY}

The considered system of the QD with a magnetic impurity is presented in Fig. 1. Conduction electrons passing through the QD interact with accumulated electrons leading to the Kondo resonance in low temperatures. A magnetic impurity is connected to the electrons in the QD by the exchange coupling $J$. The Hamiltonian corresponding to our model is written as

$$
H=\sum_{k, \sigma, \alpha} \epsilon_{k} c_{k \sigma, \alpha}^{\dagger} c_{k \sigma, \alpha}+\epsilon_{0} \sum_{\sigma} c_{0 \sigma}^{\dagger} c_{0 \sigma}+U c_{0 \uparrow}^{\dagger} c_{0 \uparrow} c_{0 \downarrow}^{\dagger} c_{0 \downarrow}+J \vec{s} \cdot \vec{S}+\sum_{k, \sigma, \alpha} t_{\alpha}\left(c_{0 \sigma}^{\dagger} c_{k \sigma, \alpha}+\text { h.c. }\right),
$$

where the first term represents the electrons in the lead $\alpha=L, R$; the second term stands for the up-most electronic level $\epsilon_{0}$ in the dot. The the third and the fourth terms describe the interactions: the Coulomb interaction of electrons with opposite spin orientation at the level $\epsilon_{0}$ and exchange interactions with the magnetic impurity. Here, $\vec{s}$ is the spin operator for electrons at the level $\epsilon_{0}$, whereas $\vec{S}$ denotes the spin operator at the magnetic impurity. It is imposed a single occupancy at the impurity $d_{\uparrow}^{\dagger} d_{\uparrow}+d_{\downarrow}^{\dagger} d_{\downarrow}=1$ and the the spin $S=1 / 2$. Therefore, $S^{z}=1 / 2\left(d_{\uparrow}^{\dagger} d_{\uparrow}-d_{\downarrow}^{\dagger} d_{\downarrow}\right)$, $S^{+}=d_{\uparrow}^{\dagger} d_{\downarrow}, S^{-}=d_{\downarrow}^{\dagger} d_{\uparrow}$, and the exchange term between the impurity and the spin of the electron localized at the QD can be written as

$$
J \vec{s} \cdot \vec{S}=\frac{1}{2} J\left(c_{0 \uparrow}^{\dagger} c_{0 \uparrow}-c_{0 \downarrow}^{\dagger} c_{0 \downarrow}\right) S^{z}+\frac{1}{2} J c_{0 \uparrow}^{\dagger} c_{0 \downarrow} S^{-}+\frac{1}{2} J c_{0 \downarrow}^{\dagger} c_{0 \uparrow} S^{+} .
$$

The last term in the Hamiltonian (1) corresponds to the coupling between the quantum dot and the leads. We are interested in specific correlations effects, so the eventual effects introduced by an asymmetric coupling are outside our purpose here. It shall be considered, for simplicity, $t_{L}=t_{R}=t$.

The energy spectrum of the isolated QD is easily to calculate (see the Appendix A). We will show that the ground states, as well as the excited states, participate in the electronic transport. The conductance peaks corresponding to the transport through the excites states are smaller, but they are high enough to be observable in an experiment. 
Our model (1) is a generalization of the single impurity Anderson model (SIAM), to which a spin-interaction term is added. Recently, Zhang et al. [30] considered also an extended SIAM model with a a one-body phenomenological spin-flip term. Their purpose was to study a different situation, namely, the electronic transport through a quantum dot with spin-orbit interactions coupled to ferromagnetic leads. In the Hamiltonian (1), the total spin is conserved (in contrast to the model used in [30]), which is important for the electronic transport.

\section{DETERMINATION OF GREEN FUNCTIONS IN EQUATION OF MOTION SCHEME}

Now, we want to determine the conductance and other physical quantities for the model described by the Hamiltonian (1). The current can be expressed by means of the non-equilibrium Green functions as [31, 32]

$$
j=\frac{2 e}{h} \Gamma \int d \omega\left[f_{L}(\omega)-f_{R}(\omega)\right]\left(-\operatorname{Im}\left\langle\left\langle c_{0 \uparrow} \mid c_{0 \uparrow}^{\dagger}\right\rangle\right\rangle\right),
$$

where $\Gamma=2 \pi t^{2} \rho$ and $\rho=1 / 2 D$ is the DOS for the square band approximation; the half-width $D$ will be taken as unity. $f_{L}$ and $f_{R}$ are the Fermi distribution functions in the left and right leads, respectively. $\left\langle\left\langle c_{0 \uparrow} \mid c_{0 \uparrow}^{\dagger}\right\rangle\right\rangle$ is the retarded single particle Green function for an electron with the spin $\sigma=\uparrow$ at the QD, which can be determined by the equation of motion (EOM). The differential conductance for the quasi-equilibrium case can be deduced from the formula (3)

$$
\mathcal{G}=\frac{2 e^{2}}{h} \Gamma \int d \omega\left(-\frac{\partial f(\omega)}{\partial \omega}\right) \operatorname{Im}\left\langle\left\langle c_{0 \uparrow} \mid c_{0 \uparrow}^{\dagger}\right\rangle\right\rangle .
$$

The equation of motion (EOM) for the energy dependent retarded Green function is given by

$$
\omega\langle\langle A \mid B\rangle\rangle=\langle\{A, B\}\rangle+\langle\langle[A, H] \mid B\rangle\rangle,
$$

where $\langle\{A, B\}\rangle$ is the thermal average of the anticommutator between the operators $\mathrm{A}$ and $\mathrm{B}$. Whenever necessary, the averages will be calculated by means of the fluctuation-dissipation theorem $\langle A B\rangle=-(1 / \pi) \int d \omega f(\omega) \operatorname{Im}\langle\langle B \mid A\rangle\rangle$, where $f(\omega)$ is the distribution function at the dot. In equilibrium, it is equal with the Fermi distribution in the leads.

To determine the current (3) one needs the single-particle Green function $\left\langle\left\langle c_{0 \sigma} \mid c_{0 \sigma}^{\dagger}\right\rangle\right\rangle$, for which the EOM is

$$
\begin{aligned}
\left(\omega-\epsilon_{0}\right)\left\langle\left\langle c_{0 \uparrow} \mid c_{0 \uparrow}^{\dagger}\right\rangle\right\rangle & =1+U\left\langle\left\langle c_{0 \uparrow} c_{0 \downarrow}^{\dagger} c_{0 \downarrow} \mid c_{0 \uparrow}^{\dagger}\right\rangle\right\rangle+\frac{1}{2} J\left\langle\left\langle c_{0 \uparrow} S^{z} \mid c_{0 \uparrow}^{\dagger}\right\rangle\right\rangle \\
& +\frac{1}{2} J\left\langle\left\langle c_{0 \downarrow} S^{-} \mid c_{0 \uparrow}^{\dagger}\right\rangle\right\rangle+2 t \sum_{k}\left\langle\left\langle c_{k \uparrow} \mid c_{0 \uparrow}^{\dagger}\right\rangle\right\rangle
\end{aligned}
$$

As usual for an interacting problem, one has also to calculate the many-particle Green functions

$$
\begin{aligned}
\left(\omega-\epsilon_{0}\right)\left\langle\left\langle c_{0 \uparrow} S^{z} \mid c_{0 \uparrow}^{\dagger}\right\rangle\right\rangle & =\left\langle S^{z}\right\rangle+\frac{1}{8} J\left\langle\left\langle c_{0 \uparrow} \mid c_{0 \uparrow}^{\dagger}\right\rangle\right\rangle+U\left\langle\left\langle c_{0 \uparrow} c_{0 \downarrow}^{\dagger} c_{0 \downarrow} S^{z} \mid c_{0 \uparrow}^{\dagger}\right\rangle\right\rangle \\
& -\frac{1}{4} J\left\langle\left\langle c_{0 \downarrow} S^{-} \mid c_{0 \uparrow}^{\dagger}\right\rangle\right\rangle+\frac{1}{2} J\left\langle\left\langle c_{0 \downarrow} c_{0 \uparrow}^{\dagger} c_{0 \uparrow} S^{-} \mid c_{0 \uparrow}^{\dagger}\right\rangle\right\rangle \\
& +2 t \sum_{k}\left\langle\left\langle c_{k \uparrow} S^{z} \mid c_{0 \uparrow}^{\dagger}\right\rangle\right\rangle \\
\left(\omega-\epsilon_{0}+\frac{1}{4} J\right)\left\langle\left\langle c_{0 \downarrow} S^{-} \mid c_{0 \uparrow}^{\dagger}\right\rangle\right\rangle & =\frac{1}{4} J\left\langle\left\langle c_{0 \uparrow} \mid c_{0 \uparrow}^{\dagger}\right\rangle\right\rangle+\left(U+\frac{1}{2} J\right)\left\langle\left\langle c_{0 \downarrow} c_{0 \uparrow}^{\dagger} c_{0 \uparrow} S^{-} \mid c_{0 \uparrow}^{\dagger}\right\rangle\right\rangle \\
& -\frac{1}{2} J\left\langle\left\langle c_{0 \uparrow} S^{z} \mid c_{0 \uparrow}^{\dagger}\right\rangle\right\rangle+J\left\langle\left\langle c_{0 \uparrow} c_{0 \downarrow}^{\dagger} c_{0 \downarrow} S^{z} \mid c_{0 \uparrow}^{\dagger}\right\rangle\right\rangle \\
& +2 t \sum_{k}\left\langle\left\langle c_{k \downarrow} S^{-} \mid c_{0 \uparrow}^{\dagger}\right\rangle\right\rangle \\
\left(\omega-\epsilon_{0}-U\right)\left\langle\left\langle c_{0 \uparrow} c_{0 \downarrow}^{\dagger} c_{0 \downarrow} \mid c_{0 \uparrow}^{\dagger}\right\rangle\right\rangle & =\left\langle c_{0 \downarrow}^{\dagger} c_{0 \downarrow}\right\rangle+\frac{1}{2} J\left\langle\left\langle c_{0 \uparrow} c_{0 \downarrow}^{\dagger} c_{0 \downarrow} S^{z} \mid c_{0 \uparrow}^{\dagger}\right\rangle\right\rangle+\frac{1}{2} J\left\langle\left\langle c_{0 \downarrow} c_{0 \uparrow}^{\dagger} c_{0 \uparrow} S^{-} \mid c_{0 \uparrow}^{\dagger}\right\rangle\right\rangle \\
& +2 t \sum_{k}\left\langle\left\langle c_{k \uparrow} c_{0 \downarrow}^{\dagger} c_{0 \downarrow} \mid c_{0 \uparrow}^{\dagger}\right\rangle\right\rangle-2 t \sum_{k}\left\langle\left\langle c_{0 \uparrow} c_{k \downarrow}^{\dagger} c_{0 \downarrow} \mid c_{0 \uparrow}^{\dagger}\right\rangle\right\rangle \\
& +2 t \sum_{k}\left\langle\left\langle c_{0 \uparrow} c_{0 \downarrow}^{\dagger} c_{k \downarrow} \mid c_{0 \uparrow}^{\dagger}\right\rangle\right\rangle
\end{aligned}
$$


and

$$
\begin{aligned}
\left(\omega-\epsilon_{0}-U\right)\left\langle\left\langle c_{0 \uparrow} c_{0 \downarrow}^{\dagger} c_{0 \downarrow} S^{z} \mid c_{0 \uparrow}^{\dagger}\right\rangle\right\rangle & =\left\langle c_{0 \downarrow}^{\dagger} c_{0 \downarrow} S^{z}\right\rangle+\frac{1}{8} J\left\langle\left\langle c_{0 \uparrow} c_{0 \downarrow}^{\dagger} c_{0 \downarrow} \mid c_{0 \uparrow}^{\dagger}\right\rangle\right\rangle+\frac{1}{4} J\left\langle\left\langle c_{0 \downarrow} c_{0 \uparrow}^{\dagger} c_{0 \uparrow} S^{-} \mid c_{0 \uparrow}^{\dagger}\right\rangle\right\rangle \\
& +2 t \sum_{k}\left\langle\left\langle c_{k \uparrow} c_{0 \downarrow}^{\dagger} c_{0 \downarrow} S^{z} \mid c_{0 \uparrow}^{\dagger}\right\rangle\right\rangle-2 t \sum_{k}\left\langle\left\langle c_{0 \uparrow} c_{k \downarrow}^{\dagger} c_{0 \downarrow} S^{z} \mid c_{0 \uparrow}^{\dagger}\right\rangle\right\rangle \\
& +2 t \sum_{k}\left\langle\left\langle c_{0 \uparrow} c_{0 \downarrow}^{\dagger} c_{k \downarrow} S^{z} \mid c_{0 \uparrow}^{\dagger}\right\rangle\right\rangle \\
\left(\omega-\epsilon_{0}-\frac{1}{4} J-U\right)\left\langle\left\langle c_{0 \downarrow} c_{0 \uparrow}^{\dagger} c_{0 \uparrow} S^{-} \mid c_{0 \uparrow}^{\dagger}\right\rangle\right\rangle & =-\left\langle c_{0 \uparrow}^{\dagger} c_{0 \downarrow} S^{-}\right\rangle+\frac{1}{4} J\left\langle\left\langle c_{0 \uparrow} c_{0 \downarrow}^{\dagger} c_{0 \downarrow} \mid c_{0 \uparrow}^{\dagger}\right\rangle\right\rangle+\frac{1}{2} J\left\langle\left\langle c_{0 \uparrow} c_{0 \downarrow}^{\dagger} c_{0 \downarrow} S^{z} \mid c_{0 \uparrow}^{\dagger}\right\rangle\right\rangle \\
& +2 t \sum_{k}\left\langle\left\langle c_{k \downarrow} c_{0 \uparrow}^{\dagger} c_{0 \uparrow} S^{-} \mid c_{0 \uparrow}^{\dagger}\right\rangle\right\rangle-2 t \sum_{k}\left\langle\left\langle c_{0 \downarrow} c_{k \uparrow}^{\dagger} c_{0 \uparrow} S^{-} \mid c_{0 \uparrow}^{\dagger}\right\rangle\right\rangle \\
& +2 t \sum_{k}\left\langle\left\langle c_{0 \downarrow} c_{0 \uparrow}^{\dagger} c_{k \uparrow} S^{-} \mid c_{0 \uparrow}^{\dagger}\right\rangle\right\rangle
\end{aligned}
$$

In the absence of a magnetic field, one can consider $\left\langle S^{z}\right\rangle=0,\left\langle n_{0 \downarrow}\right\rangle=\left\langle n_{0 \uparrow}\right\rangle=\left\langle c_{0 \uparrow}^{\dagger} c_{0 \uparrow}\right\rangle$ and $\left\langle c_{0 \downarrow}^{\dagger} c_{0 \downarrow} S^{z}\right\rangle=$ $-\frac{1}{2}\left\langle c_{0 \uparrow}^{\dagger} c_{0 \downarrow} S^{-}\right\rangle=-\frac{1}{3}\langle\vec{s} \cdot \vec{S}\rangle,\left(S^{z}\right)^{2}=\frac{1}{4}$ (see also [33] for some details on how the constriction $d_{\uparrow}^{\dagger} d_{\uparrow}+d_{\downarrow}^{\dagger} d_{\downarrow}=1$ is used).

We have got a set of six equations, Eqs.(6)-(8), for all Green functions at the quantum dot. These functions are coupled with other many-particle Green functions $\left\langle\left\langle c_{k \sigma} A \mid c_{0 \uparrow}^{\dagger}\right\rangle\right\rangle$, which describes the coupling between QD and the electrodes. The EOM procedure requires to write also the equation of motion for the new set of Green functions, which will be coupled with another new set of the higher order many-particle Green functions. In order to close the infinite series of the equations of motion, one has to decouple many-particle Green functions. The simplest approximation is

$$
2 t \sum_{k}\left\langle\left\langle c_{k, \sigma} A \mid c_{0, \sigma}^{\dagger}\right\rangle\right\rangle \approx-i \Gamma\left\langle\left\langle c_{0, \sigma} A \mid c_{0, \sigma}^{\dagger}\right\rangle\right\rangle .
$$

The approximation takes into account charge fluctuations between the electrode and QD, but it neglects the spin-flip processes and the Kondo resonance. This approximation correctly describes the limit of the Coulomb blockade and gives the same results as those obtained by Nagaoka [33] and by Meir et al [34] (for QD without impurity) in high temperatures. It is important to notice that all Green functions at QD are treated exactly, so the intra-dot correlations are fully accounted. This approximation will be used in the next section to study the high temperature properties of our model.

\section{STUDIES OF THE HIGH TEMPERATURES REGIME}

Using the approximation (9) we get a set of six linear equations, with the correlators $\left\langle n_{0 \uparrow}\right\rangle$ and $\left\langle c_{0 \uparrow}^{\dagger} c_{0 \uparrow} S^{z}\right\rangle$ as parameters. One can easily find analytical expressions for all many-particle Green functions at the QD. Next, the correlators are calculated by means of the appropriate Green functions $\left\langle n_{0 \uparrow}\right\rangle=-(1 / \pi) \int d \omega f(\omega) \operatorname{Im}\left\langle\left\langle c_{0 \uparrow} \mid c_{0 \uparrow}^{\dagger}\right\rangle\right\rangle$ and $\left\langle c_{0 \uparrow}^{\dagger} c_{0 \uparrow} S^{z}\right\rangle=-(1 / \pi) \int d \omega f(\omega) \operatorname{Im}\left\langle\left\langle c_{0 \uparrow} S^{z} \mid c_{0 \uparrow}^{\dagger}\right\rangle\right\rangle$. In order to calculate these integrals one has first to decompose the Green functions in simple fractions. The solutions are expressed by the digamma function [35], which at zero temperature, turns into the arctan function.

The main results of this section are presented in Fig.2. The upper panels show the conductance (solid curves) and the electron occupancy of the QD (dotted curves) as a function of the position of the dot energy $\epsilon_{0}$ for different parameters $\Gamma$. The lower panels show the corresponding behavior of the spin-spin correlator.

For $J>0$ - column a) - four conductance peaks are clearly seen, while for $J<0$ - column b) - there are two pronounced peaks and two much smaller peaks. To explain the origin of the peaks, let us start with a high value of $\epsilon_{0}$, which is much above the Fermi energy $E_{F}=0$. For this case the dot is empty. When $\epsilon_{0}$ is lowered (by an applied gate potential), a first electron is allowed to jump into the QD on the lowest available level. This is the singlet state, for case a) -see Appendix A- and it is located at $\epsilon_{0}=0.15$. For case b), the lowest state is the triplet state at $\epsilon_{0}=0.05$. By further lowering of $\epsilon_{0}$, the excited state energy becomes aligned with the Fermi level. It is the triplet state at $\epsilon_{0}=-0.05$, or the singlet state at $\epsilon_{0}=-0.15$, for the case a) and b) respectively. At these positions the conductance shows peaks, for which the height is smaller than for those corresponding to the ground states. We do not observe any changes of the electron occupancy at these positions.

The transmission through the excited states, where the total occupancy on the dot is constant, can be explained by the overlapping with the ground state and fluctuations of the charge distribution between levels. The electron 
has a probability to exist on the excited state, and from here it can tunnel into the leads. This explains the strong coupling-dependence of the excited states conductance. Notice a significant increase of the peaks corresponding to the transport through excited states as the coupling $\Gamma$ increases from 0.01 to 0.08 . Experimentalists indeed have measured such small conductance peaks corresponding to transport through excited states (e.g. 36, 37]), when the total occupancy on the QD is constant. Apart from describing an impurity dot, our model may be one of the simplest to allow the analysis of excited states behavior in transport.

The transmittance through the excited states is more pronounced in Fig2.a than in Fig.2b. This is because the triplet state (that is the excited state in the case a) is three-fold degenerate and in favored in transport in comparison with the non-degenerate singlet state. From the same reason, one can notice that, on the contrary, the peak corresponding to the ground state (triplet) in Fig.2b is higher than in Fig.2a (singlet). The conductance peaks corresponding to the transport through the ground states remain then practically unchanged, only a broadening effect is noticed. A detailed discussion of the peaks amplitude will be given further in this section.

We remind that the resonances in conductance appear when two quantum states with consecutive occupancy are energetically available in the same time, allowing for charge fluctuations. So, one can find the positions of the resonances with the equation $E(\nu, n)=E\left(\nu^{\prime}, n \pm 1\right)$, meaning that an energy from the spectrum with $n$ electrons becomes equal with an energy for $n \pm 1$ electrons - see Appendix A. By further lowering of $\epsilon_{0}$, the energy for double occupancy will become equal with the excited single-particle energy. This will generate a small peak in conductance, and no variation of occupancy. Finally, a big peak is noticed corresponding to the adding of the second electron. Actually, a particle-whole symmetry is easy to notice in Fig.2, and we shall focus the further discussion on the first two peaks from the right (marked by the vertical, dotted lines).

The lower panel in Fig.2 shows the evolution of the spin-spin correlator $\langle\vec{s} \cdot \vec{S}\rangle$, which is a measure of the coupling between the spin of an electron accumulated at the QD and the magnetic impurity spin. For $J>0$, electrons at the QD are antiferromagnetic coupled with the impurity spin, and therefore, the spin-spin correlator is negative. Its minimal value is $-0.7,-0.5$ and -0.3 for $\Gamma=0.01,0.04$ and 0.08 , respectively. For the case $\Gamma=0.01$, the length of the total spin, $\left\langle\vec{S}_{\text {tot }}^{2}\right\rangle=\left\langle\vec{s}^{2}\right\rangle+2\langle\vec{s} \cdot \vec{S}\rangle+\left\langle\vec{S}^{2}\right\rangle$ is then reduced to 0.1, i.e. it is almost compensated. When the triplet state gets below Fermi energy, the correlator $\langle\vec{s} \cdot \vec{S}\rangle$ increases, because the triplet state favors ferromagnetic coupling. Its maximal value is $\langle\vec{s} \cdot \vec{S}\rangle \approx-0.1$, which means $\left\langle\vec{S}_{\text {tot }}^{2}\right\rangle \approx 1.3$ close to $3 / 2$ - the value of the magnetic moment for two free $S=1 / 2$ spins - see the central part of the graph. The result is important because it shows that the excited states contribute to the formation of the local magnetic moment at the QD. It can have also influence on the Kondo singlet formation with the electrons from the leads. Section V will show that indeed this is the case.

The evolution of the spin-spin correlator for the ferromagnetic case, presented in Fig.2b, can be explained in the same way. When the triplet state crosses $E_{F}$ the spin-spin correlator reaches maximal values $0.23,0.2$ and 0.15 , for $\Gamma=0.01,0.04$ and 0.08, respectively. The total magnetic moment increases up to 1.94, 1.86 and 1.7. For each of the three situations, the spin-spin correlator and the total moment begin to decrease when the singlet state goes below $E_{F}$.

We want to discuss in more details the heights of the conductance peaks corresponding to the singlet and the triplet states. A simple analytical solution can be obtained for the conductance, for $T=0$ and in the large $U$ limit, for the singlet and triplet resonances

$$
\mathcal{G}=\mathcal{G}_{S}+\mathcal{G}_{T}=\frac{1+2 \phi_{T}}{2\left(3+4 \phi_{S}-4 \phi_{S} \phi_{T}\right)} R_{S}+\frac{3\left(1+2 \phi_{S}\right)}{2\left(3+4 \phi_{S}-4 \phi_{S} \phi_{T}\right)} R_{T},
$$

where $R_{S}=\left(2 e^{2} / h\right) \times \Gamma^{2} /\left[\Gamma^{2}+\left(E_{F}-\epsilon_{0}+3 J / 4\right)^{2}\right]$ and $R_{T}=\left(2 e^{2} / h\right) \times \Gamma^{2} /\left[\Gamma^{2}+\left(E_{F}-\epsilon_{0}-J / 4\right)^{2}\right]$ corresponds to the resonant conductance through the singlet and the triplet level, respectively, $\phi_{S}=\arctan \left[\left(\epsilon_{0}-E_{F}-3 J / 4\right) / \Gamma\right] / \pi$ and $\phi_{T}=\arctan \left[\left(\epsilon_{0}-E_{F}+J / 4\right) / \Gamma\right] / \pi$. The fractions that multiply the resonances $R_{S(T)}$ contain the information about the height of the conductance peaks. The ground and excited states role changes with the sign of $J$, and the dependence on the coupling also changes, being much more pronounced for the excited state. All these effects are incorporated in formula (10). This zero-temperature formula is given for its simplicity, but it describes well also situations like in Fig.2, where temperature $T=10^{-3}$ is considerably lower than the broadening due to coupling with the leads $(\Gamma=0.01$ to 0.08). For higher temperatures, one has to replace in formula (10): $\phi_{S}=-\operatorname{Im}\left\{\Psi\left[1 / 2-i\left(\epsilon_{0}-E_{F}-3 J / 4+i \Gamma\right) /(2 \pi T)\right]\right\} / \pi$, $\phi_{T}$ in a similar way ( $\Psi$ is the digamma function, see also [38]), and then perform an integral required by the conductance formula deduced from Eq.3. Fig.2 was plotted in this way, with the complete formula.

By employing further the simplified form Eq.(10), one can estimate the ratio between the heights of the conductance for the singlet and triplet peaks. We notice that in Eq.(10), the parameter $\phi_{S}\left(\phi_{T}\right)$ vanishes at the singlet (triplet) resonant level, and therefore, one can straightforward calculate the ratio of the conductance peaks

$$
\frac{\max \left[\mathcal{G}_{S}\right]}{\max \left[\mathcal{G}_{T}\right]}=\frac{\left(1+2 \phi_{0}\right)\left(3-4 \phi_{0}\right)}{9\left(1-2 \phi_{0}\right)}
$$


a) J>0
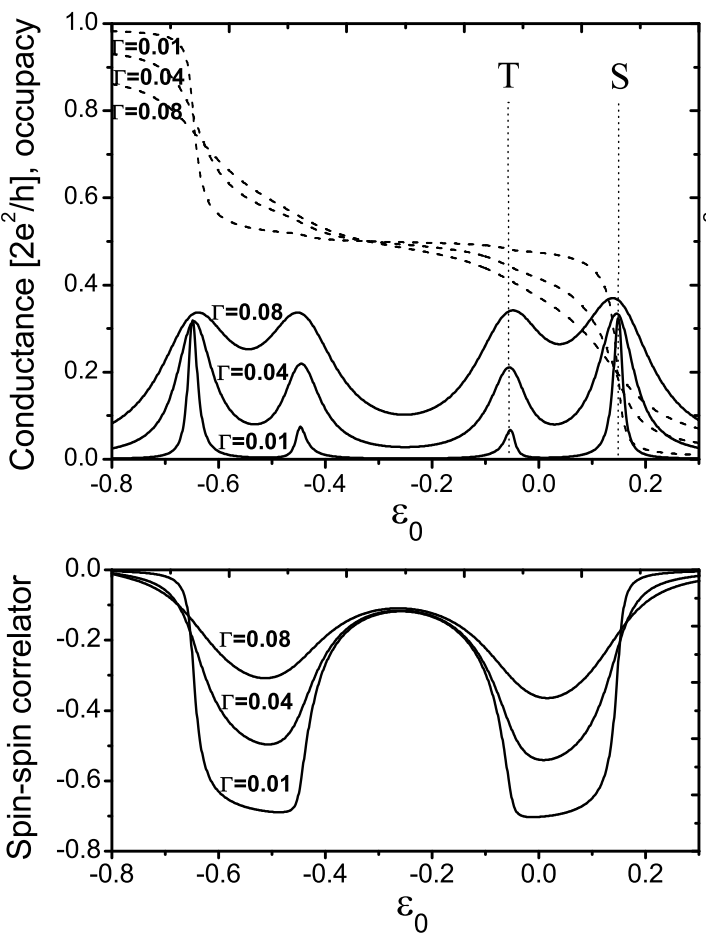

b) $\mathrm{J}<0$
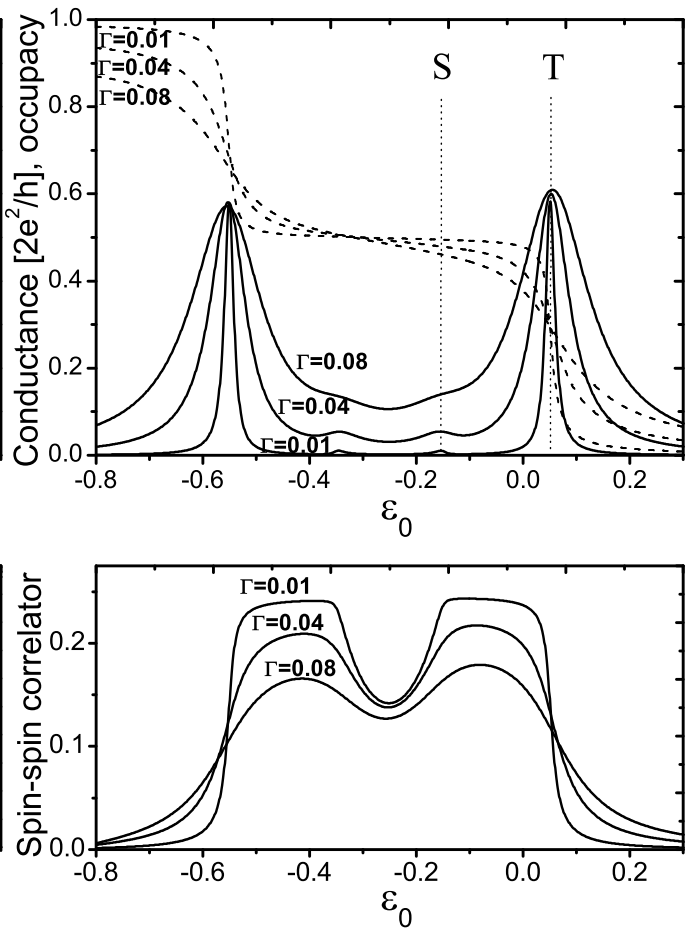

FIG. 2: The conductance (solid curves), the dot occupancy per spin $\left\langle n_{0 \sigma}\right\rangle$ (dotted curves) - upper panels, and the spin-spin correlator $\langle\vec{s} \cdot \vec{S}\rangle$ - lower panels, plotted versus the dot energy $\epsilon_{0}$, for $U=0.5,|J|=0.2, E_{F}=0, T=10^{-3}$, and for different values of $\Gamma=0.01,0.04$ and 0.08 . The vertical lines indicate the position of the Singlet and the Triplet levels.

where $\phi_{0}=\arctan (J / \Gamma) / \pi$. For the antiferromagnetic coupling the electronic transmission through the singlet state dominates and in the limit $J \gg \Gamma$ this ratio goes to infinity and the triplet peak disappears. In the case of the ferromagnetic coupling and $|J| \gg \Gamma$ we have opposite situation - the singlet states disappears from transport and only the peak corresponding to the triplet ground state is visible.

In [4, 39], the authors model a similar spin-dependent transport situation (for the QD and QPC, respectively), but the correlations and the effects of the Fermi sea are neglected. Their problem is analogous to the electron collision with a hydrogen atom (see [40]). In such a case, by averaging all possible scattering processes, the height of the singlet and triplet peaks will be proportional with their degeneracy resulting the $1 / 3$ peak height ratio - independent of the sign of $J$, also independent of the coupling to the leads. Formulas (10)-(11) are a natural generalization. The difference results from the charge accumulation and the Coulomb blockade effect.

In the final part of this section, we analyze the evolution of the phase shift for the scattered electrons. The phase problem has raised considerable interest in mesoscopic physics [41]. The principle of the phase shift experimental measurement [26, 27] uses an interference process, which has been realized by inserting QD - for which the phase is measured - in one arm of an Aharonov-Bohm interferometer. The electronic wave passing through QD acquires a phase shift and then interferes with the wave travelling through the reference arm. In this way, one can measure not only the amplitude of the transmission, but also its phase, which is extracted from the phase of the Aharonov-Bohm conductance oscillations. The authors expected a phase evolution in concordance with the Fridel sum rule, growing with $\pi$ on resonances, and with constant values between them. Instead, an intriguing universal phase-lapse effect was noticed [26, 27], meaning that the phase has a drop of $\pi$ between any pair of resonances. The aspect has not yet received a satisfactory explanation. Recently, Avinun-Kalish et al. [28] realized a similar experiment, but with a supplementary possibility to control the exact number of electrons in QD (up to 20 electrons). For the first few electrons (about 10), the phase shift had a complex non-universal behavior, with dips rather than phase-lapses, and for the next electrons, the "universal" behavior was recovered. Rontani demonstrated, in a very recent paper [29], 
that such features in the phase evolution are normal to happen, as the mesoscopic systems with electronic correlations do not obey the classical Fridel sum rule. It means that the experimentally measured phase shift is not equal to $\pi$ multiplied with the accumulated charge, but may have variations in the case of electronic correlations in the system. We explicitly prove the existence of such dips in the phase evolution, for the model discussed in this paper. Moreover, they receive a spectral interpretation, as being a fingerprint of the excited states.

For simplicity, we consider here only the situation $\Gamma=0.01$, when the singlet and triplet peaks are well-separated (but the conclusions are general). One could naively expect that the phase grows with $2 \pi$ on the two separate peaks, or that the phase evolution obeys the Fridel sum rule and accurately follows the occupancy curves. However, both hypotheses are wrong, as the ground state is decisively influencing the phase evolution on the excited state. We must remember that the very existence of the excited states peaks is attributed to the overlapping with the ground states peaks.

The phase shift can be expressed as the argument of the single-electron Green function [4, 42]

$$
\Phi=\arctan \left[\frac{\operatorname{Im}\left\langle\left\langle c_{0 \sigma} \mid c_{0 \sigma}^{\dagger}\right\rangle\right\rangle_{\omega=E_{F}}}{\operatorname{Re}\left\langle\left\langle c_{0 \sigma} \mid c_{0 \sigma}^{\dagger}\right\rangle\right\rangle_{\omega=E_{F}}}\right] .
$$

In a single-particle scattering picture, the phase defined as (12) is equal to the phase-shift of the electron wavefunction. The definition is identical with the definition of $\theta_{t}$ in [42]. The authors define the phases as the argument of the scattering matrix elements, which are the retarded Green functions.

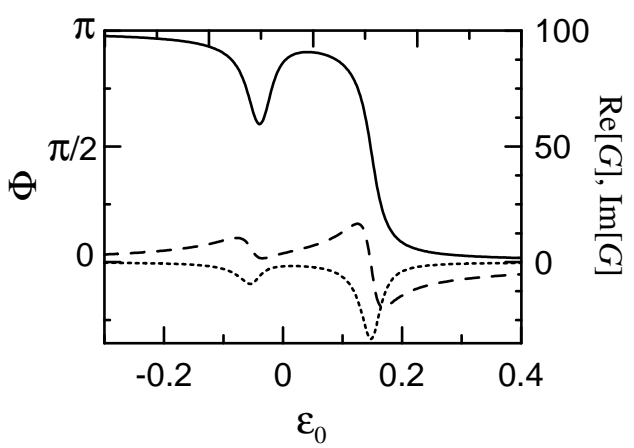

FIG. 3: Evolution of the phase shift $\Phi$ (solid curve), the real and the imaginary part of the single particle Green function $G=\left\langle\left\langle c_{0 \sigma} \mid c_{0 \sigma}^{\dagger}\right\rangle\right\rangle_{\omega=E_{F}}$ (dashed and dotted curves - in 1/D units) for the same parameters as in Fig.2a and $\Gamma=0.01$.

Fig.3 presents the phase evolution, the real and the imaginary part of the Green function. The phase evolves with $\pi$ on the peak corresponding to the ground state (around $\epsilon_{0}=0.15$ ), where an electron is added. Then the phase presents a dip on the excited state peak (around $\epsilon_{0}=-0.05$ ), in conditions of unchanged dot occupancy (see Fig.2). Notice that the real part of the Green function (the dashed curve in Fig.3) changes sign at the ground state position, but not at the excited state position, which is why the argument of the complex Green function (12) remains in the same quadrant in the latter case. The phase exactly at the triplet position is easy to calculate. We obtained the minimal value in the $\operatorname{dip}(\pi-\arctan [6 / \pi]) \simeq 0.65 \pi$ [43], for a low coupling (see Fig.3). For the negative exchange parameter (as in Fig.2, column b), and the singlet excited state, the minimal value for the dip is $(\pi-\arctan [2 /(3 \pi)]) \simeq 0.93 \pi$.

The observed dip is a signature of the excited states and can be used to probe the existence of such states through phase measurements. Also, phase measurements can, in principle, provide information about the nature of the excited states - we obtained the amplitude of the dip different for the singlet and triplet.

Our phase calculations can be correlated with the recent experiment by Avinun et al. [28], where such dips in the phase evolution were observed. As mentioned, the authors measured the transmission phase, and have reported a non-universal behavior. The measured phase showed the expected evolution with $\pi$ on the resonances, but the authors also observed a number of dips of variable amplitude (but less than $\pi$ ). Rontani [29] proved that the phase shift is not necessarily an integer of $\pi$ between two consecutive Coulomb blockade regions, if a complete formula is employed. The author stated that values lower than $\pi$ for the phase, through regions with constant occupancy, are a fingerprint of electronic correlations. The dips may even reach the value zero for the case of the spin blockade.

\section{AN INSIGHT ON THE KONDO REGIME}

In this section, we analyze the Kondo physics for our system, in a low temperature. In this regime, the electron from the dot may develop a Kondo-correlated state with the electrons in the leads. This is in competition with the 
spin-coupling with the magnetic impurity.

A study of the Kondo resonance requires a method which takes into account correlations with electrons in the leads and the virtual spin-flip processes. One has to use the higher order decoupling scheme than that one used in the previous section. The next step of EOM involves the Green functions with one operator from the leads and decoupling for the Green functions with two operators from the leads. Here, an example of EOM

$$
\begin{array}{r}
\left(\omega-\epsilon_{k}\right)\left\langle\left\langle c_{0 \uparrow} c_{0 \downarrow}^{\dagger} c_{k \downarrow} S^{z} \mid c_{0 \uparrow}^{\dagger}\right\rangle\right\rangle=\left\langle c_{0 \downarrow}^{\dagger} c_{k \downarrow} S^{z}\right\rangle+t\left\langle\left\langle c_{0 \uparrow} c_{0 \downarrow}^{\dagger} c_{0 \downarrow} S^{z} \mid c_{0 \uparrow}^{\dagger}\right\rangle\right\rangle+2 t \sum_{q}\left\langle\left\langle c_{q \uparrow} c_{0 \downarrow}^{\dagger} c_{k \downarrow} S^{z} \mid c_{0 \uparrow}^{\dagger}\right\rangle\right\rangle \\
-2 t \sum_{q}\left\langle\left\langle c_{0 \uparrow} c_{q \downarrow}^{\dagger} c_{k \downarrow} S^{z} \mid c_{0 \uparrow}^{\dagger}\right\rangle\right\rangle+\frac{1}{4} J\left\langle\left\langle c_{0 \uparrow} c_{0 \downarrow}^{\dagger} c_{k \downarrow} \mid c_{0 \uparrow}^{\dagger}\right\rangle\right\rangle+\frac{1}{4} J\left\langle\left\langle c_{k \downarrow} c_{0 \downarrow}^{\dagger} c_{0 \downarrow} S^{-} \mid c_{0 \uparrow}^{\dagger}\right\rangle\right\rangle \\
+\frac{1}{4} J\left\langle\left\langle c_{k \downarrow} c_{0 \uparrow}^{\dagger} c_{0 \uparrow} S^{-} \mid c_{0 \uparrow}^{\dagger}\right\rangle\right\rangle-\frac{1}{2} J\left\langle\left\langle c_{k \downarrow} c_{0 \uparrow}^{\dagger} c_{0 \uparrow} c_{0 \downarrow}^{\dagger} c_{0 \downarrow} S^{-} \mid c_{0 \uparrow}^{\dagger}\right\rangle\right\rangle .
\end{array}
$$

The higher order Green functions are decoupled according the following scheme

$$
\begin{aligned}
\left\langle\left\langle c_{0 \uparrow} c_{q \downarrow}^{\dagger} c_{k \downarrow} \mid c_{0 \uparrow}^{\dagger}\right\rangle\right\rangle & \approx\left\langle c_{q \downarrow}^{\dagger} c_{k \downarrow}\right\rangle\left\langle\left\langle c_{0 \uparrow} \mid c_{0 \uparrow}^{\dagger}\right\rangle\right\rangle \approx \delta_{k q} f\left(\epsilon_{k}\right)\left\langle\left\langle c_{0 \uparrow} \mid c_{0 \uparrow}^{\dagger}\right\rangle\right\rangle, \\
\left\langle\left\langle c_{0 \uparrow} c_{q \uparrow}^{\dagger} c_{k \downarrow} S^{-} \mid c_{0 \uparrow}^{\dagger}\right\rangle\right\rangle & \approx\left\langle c_{q \uparrow}^{\dagger} c_{k \downarrow} S^{-}\right\rangle\left\langle\left\langle c_{0 \uparrow} \mid c_{0 \uparrow}^{\dagger}\right\rangle\right\rangle \approx \delta_{k q} \lambda f\left(\epsilon_{k}\right)\left\langle c_{0 \uparrow}^{\dagger} c_{0 \downarrow} S^{-}\right\rangle\left\langle\left\langle c_{0 \uparrow} \mid c_{0 \uparrow}^{\dagger}\right\rangle\right\rangle, \\
\left\langle\left\langle c_{0 \uparrow} c_{q \downarrow}^{\dagger} c_{k \downarrow} S^{z} \mid c_{0 \uparrow}^{\dagger}\right\rangle\right\rangle & \approx \delta_{k q} f\left(\epsilon_{k}\right)\left\langle\left\langle c_{0 \uparrow} S^{z} \mid c_{0 \uparrow}^{\dagger}\right\rangle\right\rangle+\delta_{k q} \lambda f\left(\epsilon_{k}\right)\left\langle c_{0 \downarrow}^{\dagger} c_{0 \downarrow} S^{z}\right\rangle\left\langle\left\langle c_{0 \uparrow} \mid c_{0 \uparrow}^{\dagger}\right\rangle\right\rangle .
\end{aligned}
$$

The decoupling procedure follows that one proposed by Meir 34] (also used e.g. in [30, 44, 45]) and by Nagaoka [33] for the Green functions that also contain the localized spin operator. Only the averages that conserve the total spin are kept. The system of equations must be closed, so we need to express further the spin-spin correlator $\left\langle c_{q \uparrow}^{\dagger} c_{k \downarrow} S^{-}\right\rangle$ between the electrons in the leads and the localized spin. This is considered to be proportional to the dot spin-spin correlator, by a parameter $\lambda<1$. The choice is justified by the fact that the localized spin is only indirectly coupled to the electrons in the leads. We have checked that the main results are not qualitatively influence for any $0<\lambda<1$. In the numerical calculations we put $\lambda=t^{2} / D^{2}$.

After the decoupling scheme (14), the set of Eqs.(13) (there are 14 equations) becomes linear and is easy to solve, in the $U \rightarrow \infty$ limit. The solution for the Green functions contains the fractions: $1 /\left(\omega-\epsilon_{k}\right), 1 /\left(\omega-\epsilon_{k}+J\right)$ and $1 /\left(\omega-\epsilon_{k}-J\right)$. Next, we come back to the initial system Eqs.(6)-(8), where the summation over $k$ leads to the appearance of three digamma functions [35]

$$
F_{a}(\omega) \equiv-\sum_{k} \frac{f\left(\epsilon_{k}\right)}{\omega-\epsilon_{k}+a}=i \frac{\pi}{2}+\ln \left(\frac{2 \pi T}{D}\right)+\Psi\left(\frac{1}{2}-i \frac{\omega+a}{2 \pi T}\right)
$$

with $a=0$ and $\pm J$. Here, $\Psi$ denotes the digamma function. For $T<T_{K}$, the function $F_{a}$ has a pronounced maximum around $\omega=-a$.

Fig.4 presents the density of states (DOS) of our system for three different cases: a) when both levels, singlet and triplet, are below $\left.E_{F}, \mathrm{~b}\right)$ the triplet state is below $E_{F}$, but the singlet state is above, and c) the singlet state is below $E_{F}$ and the triplet above $E_{F}$. In the panel a), the singlet and triplet peaks overlap, due to the small $J / \Gamma$ ratio. Our model gives three peaks in the DOS: one at the Fermi level $E_{F}=0$ and two symmetrical side peaks, at $\pm J$, corresponding to the basic excitations in the system. The central peak can be associated with the three-fold degeneracy of the triplet state. We point out that in the measurements of Sasaki (see Fig.4a in [23]) such a three-peak structure can be seen in the case of the triplet ground state as well as for the singlet ground state, where the "zero" peak is however much less pronounced and the other two peaks are considerably enhanced.

For a large level spacing (large $J$ ), only the peak at $E_{F}$ is of interest. The distant side peaks would not influence the conductance in any way. Our calculations show that the central Kondo peak is formed when the triplet state is below $E_{F}$ (Fig4.b), and it is not formed when only the singlet state is below $E_{F}$ (Fig4.c). Such a result was expected because the singlet state is not degenerate, and a Kondo peak at the Fermi energy can only appear when the system has a degenerate occupied level (it is not exclusively a spin effect, but, more generally, it originates in the Pauli exclusion principle).

In Appendix B we show that the existence of the central Kondo peak can be related with the value of the spin-spin correlator. The peak is absent for a strong antiferromagnetic coupling of the spins inside the dot. Such a case happens when the singlet state is below the Fermi energy and the triplet above. Therefore, the arguments based on the value of the spin-spin correlator (given in Appendix B) lead to the same conclusion as the arguments based on degeneracy considerations (presented in the previous paragraph).

In this section we have focused exclusively on the description of the Kondo peaks in the DOS. However, the differential conductance accurately follows the DOS. In [46], the authors prove the decoupling of Meir [34] to give 


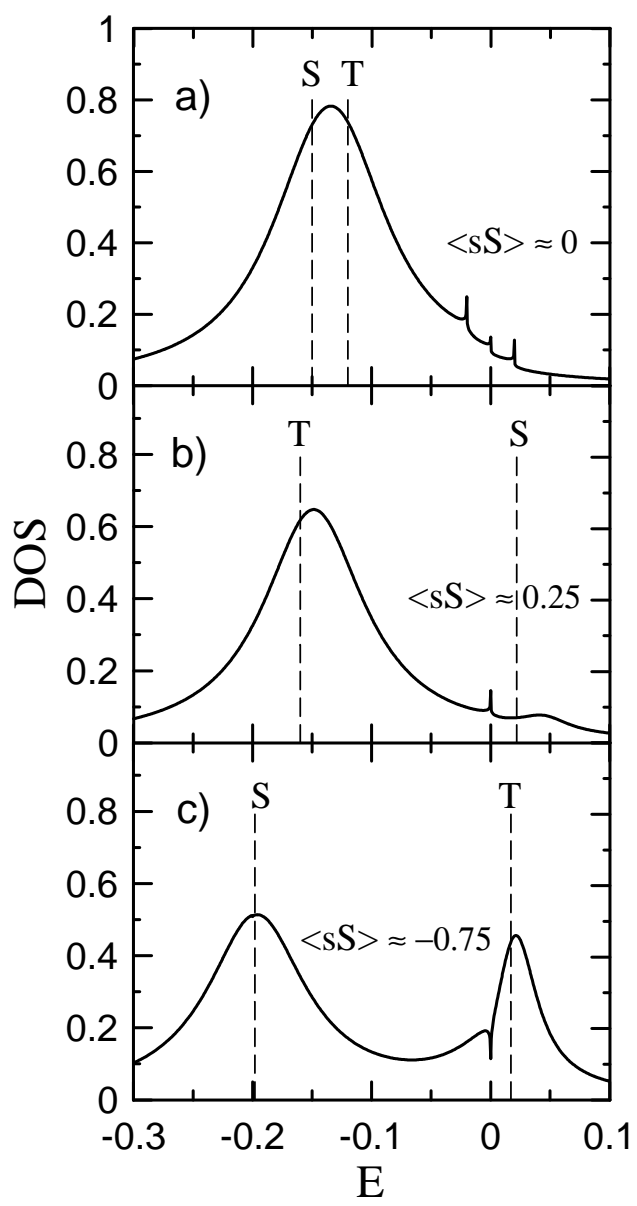

FIG. 4: Plots of the local density of states (DOS) for the Kondo regime: a) $\epsilon_{0}=-0.15$ and $\left.J=0.02 ; \mathrm{b}\right) \epsilon_{0}=-0.11$ and $J=-0.2$; c) $\epsilon_{0}=-0.05$ and $J=0.2$. The corresponding value of the spin-spin correlator is written on each graph. The vertical lines indicate the positions of the singlet and triplet levels. For all the three plots $E_{F}=0, \Gamma=0.025$ and $T=10^{-6}$.

physically correct results (qualitatively) in the Kondo regime, but the decoupling cannot be used in the mixed-valance regime - when charge fluctuations are mixed with spin fluctuations. This is why, in this section, we restricted the analysis to the spin fluctuations and the Kondo regime, when the occupancy on the dot is $\approx 1$. For the mixed-valance regime, the approximation made by Lacroix [47] for the SIAM should be used instead. A generalization of [47] was only done for systems with a complex noninteracting network around the impurity [46, 48, 49, 50, 51, 52]. In the present paper, we added an interaction term, considerably increasing the complexity, and believe that the scheme [47] may no longer be applicable. The more simple decoupling used in section IV allows the analysis of charge fluctuations, but looses the spin fluctuations - so the two decouplings presented in this paper are complementary.

\section{CONCLUSIONS}

The paper addresses the problem of electronic transport through a small quantum dot with a magnetic impurity. Our Hamiltonian model is the single impurity Anderson extended by a term of an exchange interaction between the electrons at QD and the magnetic impurity. The calculations have been performed by means of the Green functions method within the EOM scheme and presented in detail, with two different decoupling proposed, in high and low temperatures, respectively.

We have studied the charge fluctuations for high temperatures, the aspect not addressed before for the case of QDs with magnetic impurities. The singlet and triplet conductance peaks are shown to depend on the ratio $J / \Gamma$ (the exchange interaction versus the coupling strength with the leads). In particular, the transmission through the excited states depends strongly on the coupling to the leads, while the transmission through the ground states show just a broadening effect. 
The spin-spin coupling $\langle\vec{s} \cdot \vec{S}\rangle$ between the magnetic impurity and the electrons in the dot is also analyzed. The electron that enters the dot forms with the magnetic impurity a correlated singlet or a triplet ground state, respectively to the sign of the exchange parameter. The absolute value of the correlator $|\langle\vec{s} \cdot \vec{S}\rangle|$ reaches then the maximal value. When the excited states are shifted below the Fermi energy, the spin-spin coupling decreases. In the regime when the levels are dip below $E_{F}$, the spins behave like free spins and $|\langle\vec{s} \cdot \vec{S}\rangle|$ reaches its minimal value. This is due to an internal charge redistribution between the ground and the excited states - due to charge fluctuations: from the ground state to the electrode and to the excited state at QD.

The paper also brings some new insights in the phase shift problem for the transmitted electrons. We find that the electronic phase shows a specific dip at the excited state position, in conditions of constant occupancy on QD. Similar features in the phase evolution were reported in a recent quantum interference experiment performed by Avinun-Kalish et al. [28]. This experimental findings inspired Rontani 29] to prove a generalized Fridel sum rule. Our calculations are an explicit demonstration that such dips in the phase evolution are due to electronic correlations in the system, and a redistribution of charge. The phase measurement seems to be be a very good detection method for such states, in a quantum interferometer device.

In the Kondo regime, there is a competition between the spin-spin coupling with the magnetic impurity and the Kondo correlations with the electrons from the leads. This competition was studied before in a different system, namely a large isolated quantum dot, where it was found that for the exchange parameter higher than a limit value, the Kondo correlations are destroyed [3]. For the case of a double-dot system, one can tune between a local singlet or a double-Kondo situation by varying different parameters of the system, like the interdot coupling [9, 10, 11]. Our model is different, but the conclusion is in agreement: we found a limit value of the impurity-electron spin-spin correlator, that inhibits the formation of the Kondo resonance. For the case when both singlet and triplet levels are below the Fermi energy, the spins are weakly correlated and we predict three Kondo peaks in the density of states: one at $E_{F}$ and the other two at $E_{F} \pm J$, corresponding to the excitation energy.

\section{Acknowledgments}

The work was supported by the project RTNNANO contract No. MRTN-CT-2003-504574 and by in part by the Ministry of Science and Higher Education (Poland).

\section{APPENDIX A: SPECTRUM OF THE ISOLATED DOT}

The isolated dot is described by the Hamiltonian (1), if we put $t=0$. The energy spectrum is composed from the empty state, the states with one and two electrons on the level $\epsilon_{0}$. For single occupancy we have

$$
\begin{aligned}
H|\uparrow \uparrow\rangle & =\left(\epsilon_{0}+\frac{1}{4} J\right)|\uparrow \uparrow\rangle \text { (Triplet), } \\
H|\downarrow \Downarrow\rangle & =\left(\epsilon_{0}+\frac{1}{4} J\right)|\downarrow \Downarrow\rangle \text { (Triplet), } \\
H \frac{1}{\sqrt{2}}(|\uparrow \downarrow\rangle+|\downarrow \uparrow\rangle) & =\left(\epsilon_{0}+\frac{1}{4} J\right) \frac{1}{\sqrt{2}}(|\uparrow \downarrow\rangle+|\downarrow \uparrow\rangle) \text { (Triplet), } \\
H \frac{1}{\sqrt{2}}(|\uparrow \Downarrow\rangle-|\downarrow \uparrow\rangle) & =\left(\epsilon_{0}-\frac{3}{4} J\right) \frac{1}{\sqrt{2}}(|\uparrow \Downarrow\rangle-|\downarrow \uparrow\rangle) \text { (Singlet), }
\end{aligned}
$$

where $|\uparrow \uparrow\rangle$ is the state with the electron with the spin $\sigma=\uparrow$ at the state $\epsilon_{0}$ and the spin up at the impurity, etc. For double occupancy, there is a two-fold degenerate state

$$
\begin{aligned}
H|\uparrow \downarrow \uparrow\rangle & =\left(2 \epsilon_{0}+U\right)|\uparrow \downarrow \uparrow\rangle, \\
H \mid \uparrow \downarrow \downarrow \downarrow & =\left(2 \epsilon_{0}+U\right)|\uparrow \downarrow \downarrow \downarrow\rangle .
\end{aligned}
$$

For single occupancy, we see that there are two possible states. The lowest in energy will be the ground state of the system (singlet for positive $J$ and triplet for negative $J$ ), and the other will be excited state. The peaks in transmittance correspond to the poles of the single-particle Green function, which is found with the condition $\omega-E\left(\nu^{\prime}, n \pm 1\right)+E(\nu, n)=0$ at $\omega=E_{F}$, where $E(\nu, n)$ is an energy state $\nu$ from the spectrum for QD with $n$ 
electrons (see e.g. [53]). In our case, for $E_{F}=0$, this is equivalent with imposing the condition $E(\nu, n)=E\left(\nu^{\prime}, n \pm 1\right)$, meaning that an energy level $\nu$ from the spectrum with $n$ electrons becomes equal with an energy level $\nu^{\prime}$ for $n \pm 1$ electrons. This can be achieved by varying the diagonal energy $\epsilon_{0}$ with an applied gate potential. For the adding of the first electron we have the resonances at $\epsilon_{0}=3 J / 4$ and $\epsilon_{0}=-J / 4$; for the adding of the second electron we will have two resonances at $\epsilon_{0}=-U+J / 4$ and $\epsilon_{0}=-U-3 J / 4$.

\section{APPENDIX B: DISCUSSION ON THE FORMATION OF THE KONDO RESONANCE}

In order to get a deeper insight on the formation of the Kondo resonances, one can assume that, in the vicinity the singularity point $\omega=-a$, the function $F_{a}$ (Eq.15) can be a dominating quantity in the Green function formula, and an expansion in $1 / F_{a}$ can be done. To be more clear, we illustrate this idea first for the case $J=0$, when we have the single-impurity Anderson Hamiltonian (within the approximations of Meir [34], which is similar to that of Lacroix's for high temperatures [47]). We have then

$$
\begin{aligned}
& \left\langle\left\langle c_{0 \uparrow} \mid c_{0 \uparrow}^{\dagger}\right\rangle\right\rangle^{J=0}=\frac{1-\left\langle n_{0 \downarrow}\right\rangle}{\omega-\epsilon_{0}+i \Gamma+t^{2} F_{0}} \\
& \frac{1}{\left\langle\left\langle c_{0 \uparrow} \mid c_{0 \uparrow}^{\dagger}\right\rangle\right\rangle^{J=0}}=t^{2} \frac{1}{1-\left\langle n_{0 \downarrow}\right\rangle} F_{0}+\ldots
\end{aligned}
$$

If the state $\epsilon_{0}$ is deep below the Fermi level $E_{F}$, so $\left\langle n_{0 \downarrow}\right\rangle \lesssim 0.5$, it results that the coefficient in front of $F_{0}$ in the expression for $1 /\left\langle\left\langle c_{0 \uparrow} \mid c_{0 \uparrow}^{\dagger}\right\rangle\right\rangle$ is positive. It ensures the existence of the Kondo peak. If we look at the denominator of the Green function, we see that this is the condition for the " $\omega-\epsilon_{0}$ " line to intersect the logarithmical enhanced peak of $F_{0}$, and therefore, to give a peak in the density of states near $E_{F}$ (see Fig.1 and Fig.2, and their interpretations, in [47]). Of course, in the simple case $J=0$, this is seen right away, the reason why we expressed the expansion of $1 /\left\langle\left\langle c_{0 \uparrow} \mid c_{0 \uparrow}^{\dagger}\right\rangle\right\rangle$ is to compare the result with the same expansion for $J \neq 0$, when the analytical formula of $\left\langle\left\langle c_{0 \uparrow} \mid c_{0 \uparrow}^{\dagger}\right\rangle\right\rangle$ is much more complex and not so transparent (but the expansion that we mentioned gives a result easy to discuss).

In the case of finite $J$ we find

$$
\frac{1}{\left\langle\left\langle c_{0 \uparrow} \mid c_{0 \uparrow}^{\dagger}\right\rangle\right\rangle}=t^{2} \frac{1-\frac{1}{3} \lambda\langle\vec{s} \cdot \vec{S}\rangle}{1-\left\langle n_{0 \downarrow}\right\rangle+\langle\vec{s} \cdot \vec{S}\rangle} F_{0}+0\left[\frac{1}{F_{0}}\right] \ldots
$$

The sign of the coefficient in front of $F_{0}$ depends on the sign of the denominator $1-\left\langle n_{0 \downarrow}\right\rangle+\langle\vec{s} \cdot \vec{S}\rangle$, because the numerator is always positive. In the Kondo regime, we have $\left\langle n_{0 \downarrow}\right\rangle \approx 0.5$ and the denominator changes its sign for $\langle\vec{s} \cdot \vec{S}\rangle$, meaning that it is positive for $\langle\vec{s} \cdot \vec{S}\rangle>-0.5$ and negative for $\langle\vec{s} \cdot \vec{S}\rangle<-0.5$.

Three cases are presented in Fig.4: the case a) with $\langle\vec{s} \cdot \vec{S}\rangle \simeq 0$, b) $\langle\vec{s} \cdot \vec{S}\rangle \simeq 1 / 4$, and the case c) with $\langle\vec{s} \cdot \vec{S}\rangle \simeq-3 / 4$. For the case a) the coefficient in front of $F_{0}$ in the expansion (17) is positive, and DOS shows the peak at $E_{F}$. In the case b), when the triplet is below $E_{F}$ and singlet above $E_{F}$, the spin-spin average $\langle\vec{s} \cdot \vec{S}\rangle$ is positive (with a maximum value $1 / 4)$, as the triplet state favors ferromagnetic coupling. For this case again the coefficient in front of $F_{0}$ is positive. A different situation is encountered in the case c), when singlet is below $E_{F}$ and triplet is above $E_{F}$. The spin-spin average is negative and, for large $J$, it can reach the minimal value $-3 / 4$. In this case the coefficient of $F_{0}$ is negative, preventing the formation of the Kondo resonance. According to our calculations, the Kondo effect develops for $\langle\vec{s} \cdot \vec{S}\rangle>-0.5$.

[1] T. Dietl, H. Ohno, F. Matsukura, J. Cibert, and D. Ferrand, Science 287, 1019 (2000).

[2] A. O. Govorov, Phys. Rev. B 70, 035321 (2004).

[3] G. Murthy, Phys. Rev. Lett. 94, 126803 (2005).

[4] A. Aldea, M. Ţolea, J. Zittartz, Physica E 28, 191 (2005).

[5] H. B. Heersche, Z. de Groot, J. A. Folk, L. P. Kouwenhoven, H. S. J. van der Zant, A. A. Houck, J. Labaziewicz, I. L. Chuang Phys. Rev. Lett. 96, 017205 (2006).

[6] R. K. Kaul, G. Zarand, S. Chandrasekharan, D. Ullmo, H. U. Baranger, Phys. Rev. Lett. 96, 176802 (2006).

[7] J. Fernandez-Rossier, R. Aguado, cond-mat/0604437

[8] N. J. Craig, J. M. Taylor, E. A. Lester, C. M. Marcus, M. P. Hanson, and A. C. Gossard, Science 304, 565 (2004).

[9] P. Simon, R. López, and Y. Oreg, Phys. Rev. Lett. 94, 086602 (2005). 
[10] M. G. Vavilov and L. I. Glazman, Phys. Rev. Lett. 94, 086805 (2005).

[11] C.-H. Chung, W. Hofstetter, cond-mat/0607772.

[12] Luis G. G. V. Dias da Silva, N. P. Sandler, K. Ingersent, and S. E. Ulloa, Phys. Rev. Lett. 97, 096603 (2006).

[13] C. A. Büsser, E. V. Anda, A. L. Lima, M. A. Davidovich, and G. Chiappe, Phys. Rev. B 62, 9907 (2000),

[14] J. Mravlje, A. Ramsak, T. Rejec, Phys. Rev. B 73, 241305(R) (2006),

[15] P. S. Cornaglia and D. R. Grempel, Phys. Rev. B 71, 075305 (2005),

[16] R. Lopez, R. Aguado, and G. Platero, Phys. Rev. Lett. 89, 136802 (2002).

[17] A. Georges and Y. Meir, Phys. Rev. Lett. 82, 3508 (1999).

[18] T. Aono and M. Eto, Phys. Rev. B 63, 125327 (2001).

[19] M. Eto and Y. V.Nazarov, Phys. Rev. B 64, 085322 (2001).

[20] D. Giuliano and A. Tagliacozzo, Phys. Rev. Lett 84, 4677 (2000).

[21] W. Izumida, O. Sakai, and S. Tarucha, Phys. Rev. Lett. 87, 216803 (2001).

[22] A. Posazhennikova and P. Coleman, Phys. Rev. Lett. 94, 036802 (2005).

[23] S. Sasaki, S. De Franceschi, J. M. Elzerman, W. G. van der Wiel, M. Eto, S. Tarucha and L. P. Kouwenhoven, Nature 405, 764 (2000).

[24] D. Goldhaber-Gordon, H. Shtrikman, D. Mahalu, D. Abusch-Magder, U. Meirav and M. A. Kastner, Nature 391, 156 (1998) .

[25] S. M. Cronenwett, T. H. Oosterkamp, and L. P. Kouwenhoven, Science 281 (1998) 540.

[26] A. Yacoby, M. Heiblum, D. Mahalu, H. Shtrikman, Phys. Rev. Lett. 74, 4047 (1995).

[27] R. Schuster, E. Buks, M. Heiblum, D. Mahalu, V. Umansky, H. Shtrikman, Nature 385, 417 (1997).

[28] M. Avinun-Kalish, M. Heiblum, O. Zarchin, D. Mahalu, and V. Umanski, Nature 436, 529 (2005).

[29] M. Rontani, Phys. Rev. Lett. 97, 076801 (2006).

[30] P. Zhang, Q.-K. Xue, Y. P. Wang, and X. C. Xie, Phys. Rev. Lett. 89, 286803 (2002).

[31] Y.Meir, N.S. Wingreen, Phys. Rev. Lett. 682512 (1992).

[32] H. Haug, A.-P. Jauho, Quantum Kinetics in Transport and Optics (Springer-Verlag, Berlin, 1998).

[33] Y. Nagaoka, Phys. Rev. 138, A1112 (1965).

[34] Y. Meir, N. S. Wingreen, and P. A. Lee, Phys. Rev. Lett. 66, 3048 (1991).

[35] D. R. Hamann, Phys. Rev 158, 570 (1967).

[36] M. C. Rogge, B. Harke, C. Fricke, F. Hohls, M. Reinwald, W. Wegscheider, and R.J. Haug, Phys. Rev. B 72, 233402 (2005).

[37] M. Sigrist, T. Ihn, K. Ensslin, M. Reinwald, and W. Wegscheider, Phys. Rev. Lett. 98, 036805 (2007).

[38] B. R. Bułka and T. Kostyrko, Phys. Rev. B 70, 205333 (2004).

[39] T. Rejec, A. Ramsak, and J.H. Jefferson, Phys. Rev. B 67, 075311 (2003).

[40] L. D. Landau and E. M. Lifshitz, Quantum Mechanics, Pergamon, Oxford, 1977; J. R. Oppenheimer, Phys. Rev. 32, 361 (1928); N. F. Mott, Proc. R. Soc. London, Ser. A 126, 259 (1930).

[41] G. Hackenbroich, Phys. Rep. 343, 463 (2001).

[42] A. L. Yeyati and M. Buttiker, Phys. Rev. B 62, 7307 (2000).

[43] For angles greater than $\pi / 2$, we should compensate the artificial $\pi$ drop from a definition of arctan. One should compute $(\pi-\arctan )$ for the value of the phase.

[44] Y. Meir, N. S. Wingreen, P. A. Lee, Phys. Rev. Lett. 70, 2601 (1993).

[45] Q.-F. Sun and H. Guo, Phys. Rev. B 64, 153306(R) (2001).

[46] V. Kashcheyevs, A. Aharony, and O. Entin-Wohlman, Phys. Rev. B 73, 125338 (2006).

[47] C. Lacroix, J. Phys. F: Metal Phys. 11, 2389 (1981)

[48] B. R. Bułka and P. Stefański, Phys. Rev. Lett. 86, 5128 (2001).

[49] O. Entin-Wohlman, A. Aharony, and Y. Meir, Phys. Rev. B 71, 035333 (2005).

[50] W. Hofstetter, J. König, and H. Schoeller, Phys. Rev. Lett. 87, 156803 (2001).

[51] P. Stefański, A. Tagliacozzo, and B. R. Bułka Phys. Rev. Lett. 93, 186805 (2004).

[52] P. Stefański, Solid State Comm. 128, 29 (2003).

[53] H. Bruus and K. Flensberg, Many-Body Quantum Theory in Consed Matter Physics, Oxford Univ. Press. (2004). 